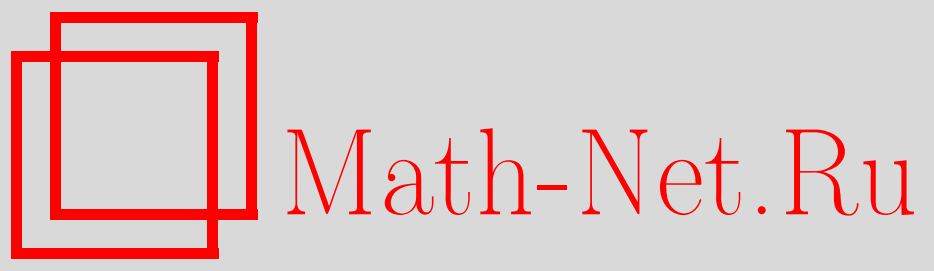

В. В. Варламов, Групповое усреднение для свободных полей в терминах гиперсферических функций над группой де Ситтера, ТМФ, 2010, том 164, номер 3, 473-480

DOI: https://doi.org/10.4213/tmf6559

Использование Общероссийского математического портала Math-Net.Ru подразумевает, что вы прочитали и согласны с пользовательским соглашением http://www.mathnet.ru/rus/agreement

Параметры загрузки:

IP: 54.198 .187 .58

26 апреля 2023 г., 15:43:49

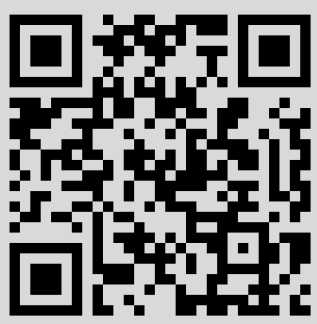




\section{ГРУППОВОЕ УСРЕДНЕНИЕ ДЛЯ СВОБОДНЫХ ПОЛЕЙ В ТЕРМИНАХ ГИПЕРСФЕРИЧЕСКИХ ФУНКЦИЙ НАД ГРУППОЙ ДЕ СИТТЕРА}

Исследуется сходимость внутренних произведений свободных полей над однородными пространствами группы де Ситтера. Показано, что в случае $N$-частичных состояний сходимость внутренних произведений определяется асимптотическим поведением гипергеометрических функций. Приводится подробное вычисление внутреннего произведения для двухчастичных состояний над четырехмерным гиперболоидом.

Ключевые слова: группа де Ситтера, групповое усреднение, внутренние произведения, гиперсферические функции, однородные пространства.

\section{1. ВВЕДЕНИЕ}

Как известно, процедура квантования систем со связями, введенная Дираком [1], в настоящее время является предметом интенсивного исследования в области теоретической физики. В подходе Дирака связи рассматриваются как операторы, действующие на векторы некоторого гильбертова пространства, а также как условия, выделяющие определенные “физические состояния", из которых затем формируется "физическое" гильбертово пространство состояний квантовой системы. Однако данная процедура квантования содержит ряд нерешенных проблем, связанных прежде всего со структурой гильбертова пространства физических состояний и с определением внутреннего произведения этих состояний. Некоторый прогресс в данном направлении был достигнут в таких подходах как БРСТ-метод [2], метод геометрического квантования [3], квантование когерентных состояний [4], методы $C^{*}$-алгебр [5], метод алгебраического квантования [6], а также метод усовершенствованного алгебраического квантования [7], [8], использующийся в работах по индукции Риффеля [9], [10] и некоторых других работах (см., например, [11]). В рамках метода усовершенствованного алгебраического квантования внутреннее произведение состояний определяется посредством техники группового усреднения. В групповом

${ }^{*}$ Сибирский государственный индустриальный университет, Новокузнецк, Россия. E-mail: vadim.varlamov@mail.ru 
усреднении используется интеграл

$$
\int_{G}\left\langle\phi_{1}|U(g)| \phi_{2}\right\rangle d g
$$

над калибровочной группой $G$, где $d g$ - так называемая “симметричная" мера Хаара на группе $G, U(g)$ - представление группы $G, \phi_{1}$ и $\phi_{2}$ - векторы состояний, принадлежащие вспомогательному гильбертову пространству $\mathcal{H}_{\text {aux }}$. Сходящееся групповое усреднение дает алгоритм для построения полного множества наблюдаемых квантовой системы [12]-[15].

В настоящей работе рассматриваются внутренние произведения для свободных полей произвольного спина над однородными пространствами группы де Ситтера $S O_{0}(1,4)$. Ключевым моментом в исследовании сходимости группового усреднения является метод определения матричных элементов представлений $U(g)$ группы $S O_{0}(1,4)$ посредством теоремы сложения для обобщенных сферических функций, ранее развитый в работах [16]-[19]. Главным преимуществом данного способа определения матричных элементов является явная факторизация матричного элемента (согласно разложению Картана) относительно подгрупп, входящих в исходную группу. Так, для группы $S O_{0}(1,4)$ матричные элементы могут быть факторизованы как относительно $S O(4)$ (максимальная компактная подгруппа), так и относительно $S O_{0}(1,3)$ (группа Лоренца). Данная факторизация позволяет разделить переменные в интеграле, задающем групповое усреднение для внутреннего произведения, т.е. вычислить по отдельности интегралы по компактным и по некомпактным подгруппам. В качестве примера вычисляется внутреннее произведение для двухчастичного случая над четырехмерным гиперболоидом. Показано, что сходимость внутреннего произведения определяется асимптотическим поведением гипергеометрических функций.

\section{2. ГРУППОВОЕ УСРЕДНЕНИЕ И ВНУТРЕННИЕ ПРОИЗВЕДЕНИЯ НАД ГРУППОЙ ДЕ СИТТЕРА}

Главной идеей в подходе Дирака является наложение на волновую функцию $|\Psi\rangle$ дополнительных условий

$$
\hat{\Lambda}_{a}^{+}|\Psi\rangle=0, \quad a=\overline{1, M},
$$

где $\hat{\Lambda}_{a}^{+}$- квантовые аналоги связей. При этом выполняются следующие коммутационные соотношения:

$$
\left[\hat{\Lambda}_{a}^{+}, \hat{\Lambda}_{b}^{+}\right]=i\left(U_{a b}^{c}\right) \hat{\Lambda}_{c}^{+},
$$

где операторы $U_{a b}^{c}$ обычно называются структурными функциями. Наиболее трудную проблему в подходе Дирака представляет построение внутреннего произведения, поскольку $\boldsymbol{\Psi}(q)$ - распределения вероятностей, функции распеределения которых в общем случае не являются квадратично-интегрируемыми функциями.

Альтернативным методом в квантовой теории является усовершенствованное алгебраическое квантование. Суть этого метода заключается во введении произвольных функций $\boldsymbol{\Phi}(q) \in \mathcal{H}_{\text {aux }}$, называемых вспомогательными векторами состояний, для которых внутреннее произведение определяется формулой $(\boldsymbol{\Phi}, \eta \boldsymbol{\Phi})$, где отображение $\eta$ таково, что $|\boldsymbol{\Psi}\rangle=\eta|\boldsymbol{\Phi}\rangle$. Дираковы состояния $|\boldsymbol{\Psi}\rangle$ образуют физическое 
гильбертово пространство $\mathcal{H}_{\text {phys }}$. В случае общей замкнутой алгебры внутреннее произведение выражается посредством интеграла над калибровочной группой с помощью формуль группового усреднения

$$
\int d_{R} g(\operatorname{det} \operatorname{Ad}\{g\})^{-1 / 2}\langle\boldsymbol{\Phi}|\check{T}(g)| \boldsymbol{\Phi}\rangle,
$$

где $d_{R} g$ - право-инвариантная мера Хаара на группе $G, \check{T}(g)$ - представление группы $G$. В случае группы де Ситтера $S O_{0}(1,4)$ имеем следующее внутреннее произведение:

$$
\left\langle\boldsymbol{\Psi}_{1} \mid \boldsymbol{\Psi}_{2}\right\rangle=\int_{g \in G} d g\left\langle\boldsymbol{\Phi}_{1}|U(g)| \mathbf{\Phi}_{2}\right\rangle .
$$

Сходимость последнего интеграла полностью определяется матричными элементами неприводимых унитарных представлений группы $S O_{0}(1,4)$.

В работе [16] было показано, что матричные элементы и сферические функции неприводимых представлений группы де Ситтера $S O_{0}(1,4)$ формулируются в рамках универсального накрытия $\operatorname{Spin}_{+}(1,4) \simeq \operatorname{Sp}(1,1)$ группы $S O_{0}(1,4)$. Сферические функции на группе $S O_{0}(1,4)$ понимаются как функции представлений класса 1 , реализуемых на однородных пространствах группы $S O_{0}(1,4)$. Список однородных пространств группы $S O_{0}(1,4)$, включая симметрические римановы и неримановы пространства, приведен в работе [16]. Так, матричные элементы, реализуемые на групповом многообразии $\mathfrak{S}_{10}$ группы $S O_{0}(1,4)$, имеют вид

$$
\mathfrak{M}_{m n}^{l}(\mathfrak{q})=e^{-i m \varphi^{q}} \mathfrak{Z}_{m n}^{l}\left(\cos \theta^{q}\right) e^{-i n \psi^{q}},
$$

где $l=0,1 / 2,1, \ldots, \mathrm{a}-l \leqslant m, n \geqslant l$. Гиперсферическая функция $\mathfrak{Z}_{m n}^{l}\left(\cos \theta^{q}\right)$ выражается в виде ряда по произведениям трех гипергеометрических функций:

$$
\begin{aligned}
& \mathfrak{Z}_{m n}^{l}\left(\cos \theta^{q}\right)=\sqrt{\frac{\Gamma(l+m+1) \Gamma(l-n+1)}{\Gamma(l-m+1) \Gamma(l+n+1)}} \cos ^{2 l} \frac{\theta}{2} \cos ^{2 l} \frac{\phi}{2} \operatorname{ch}^{2 l} \frac{\tau}{2} \times \\
& \quad \times \sum_{k=-l}^{l} \sum_{t=-l}^{l} i^{m-k} \operatorname{tg}^{m-t} \frac{\theta}{2} \operatorname{tg}^{t-k} \frac{\phi}{2} \operatorname{th}^{k-n} \frac{\tau}{2} \times \\
& \quad \times{ }_{2} F_{1}\left(\begin{array}{c}
m-l,-t-l \\
m-t+1
\end{array} \mid-\operatorname{tg}^{2} \frac{\theta}{2}\right){ }_{2} F_{1}\left(\begin{array}{c}
t-l,-k-l \\
t-k+1
\end{array} \mid-\operatorname{tg}^{2} \frac{\phi}{2}\right){ }_{2} F_{1}\left(\begin{array}{c}
k-l,-n-l \\
k-n+1
\end{array} \mid \operatorname{th}^{2} \frac{\tau}{2}\right)
\end{aligned}
$$

при $m \geqslant t, t \geqslant k, k \geqslant n$. А также существуют еще семь выражений гипергеометрического типа для функций $\mathfrak{Z}_{m n}^{l}\left(\cos \theta^{q}\right)$ при следующих значениях индексов: $m \geqslant t$, $k \geqslant t, k \geqslant n ; t \geqslant m, k \geqslant t, n \geqslant k ; t \geqslant m, t \geqslant k, n \geqslant k ; t \geqslant m, k \geqslant t, k \geqslant n ; t \geqslant m$, $t \geqslant k, k \geqslant n ; m \geqslant t, t \geqslant k, n \geqslant k ; m \geqslant t, k \geqslant t, n \geqslant k$.

\section{3. СХОДИМОСТЬ ВНУТРЕННИХ ПРОИЗВЕДЕНИЙ}

Возвращаясь к формуле группового усреднения, видим, что сходимость внутреннего произведения

$$
\left\langle\boldsymbol{\Psi}_{1} \mid \boldsymbol{\Psi}_{2}\right\rangle:=\int_{g \in G} d g\left\langle\phi_{1}|U(g)| \phi_{2}\right\rangle
$$


зависит от матричных элементов неприводимых представлений $U(g)$ группы де Ситтера $S O_{0}(1,4)$. Как было показано ранее [16], такие матричные элементы определяются посредством гиперсферической функции вида

$$
\mathfrak{M}_{m n}^{l}(\mathfrak{q})=e^{-i\left(m \varphi^{q}+n \psi^{q}\right)} \sum_{k=-l}^{l} \sum_{t=-l}^{l} P_{m k}^{l}(\cos \phi) P_{k t}^{l}(\cos \theta) \mathfrak{P}_{t n}^{l}(\operatorname{ch} \tau),
$$

где $P_{m k}^{l}(\cos \phi), P_{k t}^{l}(\cos \theta)$ - сферические функции подгруппы $S U(2)$, a $\mathfrak{P}_{t n}^{l}(\operatorname{ch} \tau)-$ сферическая функция подгруппы $S U(1,1)$. Данное выражение непосредственно следует из разложения Картана $U(g)=A^{q} K^{q} A^{q}$, где $K^{q}=S U(2) \otimes S U(2)$ и $A^{q}-$ максимальная компактная и коммутативная подгруппы группы $\operatorname{Sp}(1,1)$. С учетом этого выражения усредненное по группе внутреннее произведение (1) на групповом многообразии $\mathfrak{S}_{10}$ примет вид

$$
\left\langle\boldsymbol{\Psi}_{1} \mid \boldsymbol{\Psi}_{2}\right\rangle:=\int_{0}^{\infty} d \tau d \epsilon d \varepsilon d \omega \sin \theta^{q} e^{-m \epsilon-n(\varepsilon+\omega)}\left\langle\psi_{1}\left|P_{0} K^{q} P_{0}\right| \psi_{2}\right\rangle,
$$

где $P_{0}$ - проекторы на $S U(2)$-инвариантные состояния. Поскольку $S U(2)$ является компактной группой, то операторы $P_{0}$ не влияют на сходимость усредненного по группе внутреннего произведения. Для $N$-частичных состояний, в которых каждая частица занимает определенную моду, получим

$$
\left\langle\boldsymbol{\Psi}_{1} \mid \boldsymbol{\Psi}_{2}\right\rangle:=\int_{0}^{\infty} d \tau d \epsilon d \varepsilon d \omega \sin \theta^{q} e^{-m \epsilon-n(\varepsilon+\omega)} \mathfrak{P}_{m_{1} n_{1}}^{l}(\operatorname{ch} \tau) \ldots \mathfrak{P}_{m_{N} n_{N}}^{l}(\operatorname{ch} \tau)
$$

Сходимость последнего интеграла следует из асимптотического поведения функций гипергеометрического типа

$$
\begin{aligned}
\mathfrak{P}_{m n}^{l}(\operatorname{ch} \tau) & =\frac{1}{\Gamma(m-n+1)} \sqrt{\frac{\Gamma(l-n+1) \Gamma(l+m+1)}{\Gamma(l-m+1) \Gamma(l+n+1)}} \times \\
& \times \operatorname{ch}^{m+n} \frac{\tau}{2} \operatorname{sh}^{m-n} \frac{\tau}{2}{ }_{2} F_{1}\left(\begin{array}{c}
l+m+1, m-l \\
m-n+1
\end{array} \mid-\operatorname{sh}^{2} \frac{\tau}{2}\right) .
\end{aligned}
$$

В качестве примера рассмотрим внутреннее произведение для двухчастичных состояний на однородном пространстве $\mathcal{M}_{4}=S O_{0}(1,4) / S O(4)$. Это пространство гомеоморфно двуполостному четырехмерному гиперболоиду $H^{4}$. Следует отметить, что четырехмерное пространство Лобачевского $\mathcal{L}^{4}$, называемое также пространством де Cuттера, реализуется на гиперболоиде $H^{4}$. Сферические функции, определяемые на однородном пространстве $\mathcal{M}_{4}=H_{+}^{4} \sim S O_{0}(1,4) / S O(4)$, т.е. на верхнем листе гиперболоида $x_{0}^{2}-x_{1}^{2}-x_{2}^{2}-x_{3}^{2}-x_{4}^{2}=1$, имеют вид [16]

$$
\mathfrak{M}_{m n}^{l}(\epsilon, \tau, \varepsilon, \omega)=e^{-m \epsilon} \mathfrak{P}_{m n}^{l}(\operatorname{ch} \tau) e^{-n(\varepsilon+\omega)} \text {. }
$$

Итак, в двухчастичном случае имеем следующее внутреннее произведение:

$$
\left\langle\Psi_{1} \mid \Psi_{2}\right\rangle=\int_{0}^{\infty} d \tau d \epsilon d \varepsilon d \omega \operatorname{sh} \tau e^{-m \epsilon-n(\varepsilon+\omega)} \mathfrak{P}_{m_{1} n_{1}}^{l}(\operatorname{ch} \tau) \mathfrak{P}_{m_{2} n_{2}}^{l}(\operatorname{ch} \tau) .
$$


Очевидно, что интеграл

$$
I_{1}=\int_{0}^{\infty} d \epsilon d \varepsilon d \omega e^{-m \epsilon-n(\varepsilon+\omega)}
$$

сходится. Вычислим интеграл

$$
I_{2}=\int_{0}^{\infty} \operatorname{sh} \tau \mathfrak{P}_{m_{1} n_{1}}^{l}(\operatorname{ch} \tau) \mathfrak{P}_{m_{2} n_{2}}^{l}(\operatorname{ch} \tau) d \tau .
$$

Используя формулу (2), выразим функции $\mathfrak{P}_{m_{1} n_{1}}^{l}(\operatorname{ch} \tau)$ и $\mathfrak{P}_{m_{2} n_{2}}^{l}(\operatorname{ch} \tau)$ через гипергеометрические функции. Тогда

$$
\begin{aligned}
I_{2}= & \frac{1}{\Gamma\left(m_{1}-n_{1}+1\right) \Gamma\left(m_{2}-n_{2}+1\right)} \times \\
& \times \sqrt{\frac{\Gamma\left(l-n_{1}+1\right) \Gamma\left(l+m_{1}+1\right) \Gamma\left(l-n_{2}+1\right) \Gamma\left(l+m_{2}+1\right)}{\Gamma\left(l-m_{1}+1\right) \Gamma\left(l+n_{1}+1\right) \Gamma\left(l-m_{2}+1\right) \Gamma\left(l+n_{2}+1\right)}} \times \\
& \times \int_{0}^{\infty} \operatorname{ch}^{m_{1}+m_{2}+n_{1}+n_{2}} \frac{\tau}{2} \operatorname{sh}^{m_{1}+m_{2}-n_{1}-n_{2}} \frac{\tau}{2}{ }_{2} F_{1}\left(\begin{array}{c}
l+m_{1}+1, m_{1}-l \\
m_{1}-n_{1}+1
\end{array} \mid-\operatorname{sh}^{2} \frac{\tau}{2}\right) \times \\
& \times{ }_{2} F_{1}\left(\begin{array}{c}
l+m_{2}+1, m_{2}-l \\
m_{2}-n_{2}+1
\end{array} \mid-\operatorname{sh}^{2} \frac{\tau}{2}\right) \operatorname{sh} \tau d \tau .
\end{aligned}
$$

Первая функция $\mathfrak{P}_{m_{1} n_{1}}^{l}(\operatorname{ch} \tau)$ может быть переписана в виде

$$
\begin{aligned}
\mathfrak{P}_{m_{1} n_{1}}^{l}(\operatorname{ch} \tau)= & \sqrt{\frac{\Gamma\left(l-m_{1}+1\right) \Gamma\left(l-n_{1}+1\right)}{\Gamma\left(l+m_{1}+1\right) \Gamma\left(l+n_{1}+1\right)}} \operatorname{ch}^{m_{1}+n_{1}} \frac{\tau}{2} \times \\
& \times \operatorname{sh}^{m_{1}-n_{1}} \frac{\tau}{2} \sum_{s=0}^{l-m_{1}} \frac{(-1)^{s} \Gamma\left(l+m_{1}+s+1\right) \operatorname{sh}^{2 s}(\tau / 2)}{\Gamma(s+1) \Gamma\left(m_{1}-n_{1}+s+1\right) \Gamma\left(l-m_{1}-s+1\right)}
\end{aligned}
$$

Учитывая (5), запишем интеграл (4) следующим образом:

$$
\begin{aligned}
I_{2}= & \frac{1}{\Gamma\left(m_{2}-n_{2}+1\right)} \sqrt{\frac{\Gamma\left(l-m_{1}+1\right) \Gamma\left(l-n_{1}+1\right) \Gamma\left(l-n_{2}+1\right) \Gamma\left(l+m_{2}+1\right)}{\Gamma\left(l+m_{1}+1\right) \Gamma\left(l+n_{1}+1\right) \Gamma\left(l-m_{2}+1\right) \Gamma\left(l+n_{2}+1\right)}} \times \\
& \times \sum_{s=0}^{l-m_{1}} \frac{(-1)^{s} \Gamma\left(l+m_{1}+s+1\right)}{\Gamma(s+1) \Gamma\left(m_{1}-n_{1}+s+1\right) \Gamma\left(l-m_{1}-s+1\right)} \times \\
& \times \int_{0}^{\infty} \operatorname{ch}^{m_{1}+m_{2}+n_{1}+n_{2}} \frac{\tau}{2} \operatorname{sh}^{m_{1}+m_{2}-n_{1}-n_{2}+2 s} \frac{\tau}{2} \times \\
& \times{ }_{2} F_{1}\left(\begin{array}{c}
l+m_{2}+1, m_{2}-l \mid-\operatorname{sh}^{2} \frac{\tau}{2} \\
m_{2}-n_{2}+1
\end{array}\right) \operatorname{sh} \tau d \tau .
\end{aligned}
$$

Сделаем подстановку $z=\operatorname{ch} \tau$ в интеграле

$$
\begin{aligned}
I_{3}= & \int_{0}^{\infty} \operatorname{ch}^{m_{1}+m_{2}+n_{1}+n_{2}} \frac{\tau}{2} \operatorname{sh}^{m_{1}+m_{2}-n_{1}-n_{2}+2 s} \frac{\tau}{2} \times \\
& \times{ }_{2} F_{1}\left(\begin{array}{c}
l+m_{2}+1, m_{2}-l \\
m_{2}-n_{2}+1
\end{array} \mid-\operatorname{sh}^{2} \frac{\tau}{2}\right) \operatorname{sh} \tau d \tau
\end{aligned}
$$


имеем

$$
\begin{aligned}
I_{3}= & \int_{1}^{\infty}\left(\frac{z^{2}-1}{4}\right)^{m_{1}+m_{2}}\left(\frac{z+1}{z-1}\right)^{n_{1}+n_{2}} \times \\
& \times\left(\frac{z-1}{2}\right)^{s}{ }_{2} F_{1}\left(\begin{array}{c}
l+m_{2}+1, m_{2}-l \\
m_{2}-n_{2}+1
\end{array} \mid-\frac{z-1}{2}\right) d z .
\end{aligned}
$$

Далее, вводя новую переменную $t=-(z-1) / 2$, получим

$$
\begin{aligned}
I_{3}= & (-1)^{m_{1}+m_{2}+n_{1}+n_{2}+s+1} \times \\
& \times \int_{0}^{\infty}(1-t)^{m_{1}+m_{2}+n_{1}+n_{2}} t^{m_{1}+m_{2}+s-n_{1}-n_{2}}{ }_{2} F_{1}\left(\begin{array}{c}
l+m_{2}+1, m_{2}-l \\
m_{2}-n_{2}+1
\end{array} \mid t\right) d t .
\end{aligned}
$$

Разложим $(1-t)^{m_{1}+m_{2}+n_{1}+n_{2}}$ по формуле бинома Ньютона:

$$
\begin{aligned}
I_{3}= & \sum_{p=0}^{m_{1}+m_{2}+n_{1}+n_{2}}(-1)^{m_{1}+m_{2}+n_{1}+n_{2}+s+p+1} \frac{\left(m_{1}+m_{2}+n_{1}+n_{2}\right) !}{p !\left(m_{1}+m_{2}+n_{1}+n_{2}-p\right) !} \times \\
& \times \int_{0}^{\infty} t^{m_{1}+m_{2}+s+p-n_{1}-n_{2}}{ }_{2} F_{1}\left(\begin{array}{c}
l+m_{2}+1, m_{2}-l \\
m_{2}-n_{2}+1
\end{array} \mid t\right) d t .
\end{aligned}
$$

Чтобы вычислить последний интеграл, будем использовать формулу [20]

$$
\begin{aligned}
I_{4}= & \int t_{2}^{n} F_{1}\left(\begin{array}{c}
a, b \\
c
\end{array} \mid t\right) d t= \\
& =n ! \sum_{k=1}^{n+1}(-1)^{k+1} \frac{(c-k)_{k} t^{n-k+1}}{(a-k+1) !(a-k)_{k}(b-k)_{k}}{ }_{2} F_{1}\left(\begin{array}{c}
a-k, b-k \\
c-k
\end{array} \mid t\right) .
\end{aligned}
$$

Тогда

$$
\begin{aligned}
I_{3}= & \sum_{p=0}^{m_{1}+m_{2}+n_{1}+n_{2}} \sum_{k=1}^{m_{1}+m_{2}+s+p-n_{1}-n_{2}+1}(-1)^{m_{1}+m_{2}+n_{1}+n_{2}+s+p+k} \times \\
& \times\left(m_{1}+m_{2}+s+p-n_{1}-n_{2}\right) ! \times \\
& \times \frac{\left(m_{1}+m_{2}+n_{1}+n_{2}\right) !\left(m_{2}-n_{2}-k+1\right)_{k}}{\left(l+m_{1}-k+2\right) !\left(l+m_{2}-k+1\right)_{k}\left(m_{2}-l-k\right)_{k}} \times \\
& \times t^{m_{1}+m_{2}+s+p-n_{1}-n_{2}-k+1}{ }_{2} F_{1}\left(\begin{array}{c}
l+m_{2}-k+1, m_{2}-l-k \\
m_{2}-n_{2}-k+1
\end{array} \mid t\right) .
\end{aligned}
$$

C учетом (6) получим для двухчастичного внутреннего произведения следующее выражение:

$$
\begin{aligned}
\left\langle\boldsymbol{\Psi}_{1} \mid \boldsymbol{\Psi}_{2}\right\rangle= & \frac{1}{\Gamma\left(m_{2}-n_{2}+1\right)} \sqrt{\frac{\Gamma\left(l-m_{1}+1\right) \Gamma\left(l-n_{1}+1\right) \Gamma\left(l-n_{2}+1\right) \Gamma\left(l+m_{2}+1\right)}{\Gamma\left(l+m_{1}+1\right) \Gamma\left(l+n_{1}+1\right) \Gamma\left(l-m_{2}+1\right) \Gamma\left(l+n_{2}+1\right)}} \times \\
& \times \sum_{s=0}^{l-m_{1}} \sum_{p=0}^{m_{1}+m_{2}+n_{1}+n_{2}} \sum_{k=1}^{m_{1}+m_{2}+s+p-n_{1}-n_{2}+1}(-1)^{m_{1}+m_{2}+n_{1}+n_{2}+p+k} \times
\end{aligned}
$$




$$
\begin{aligned}
& \times \frac{\Gamma\left(l+m_{1}+s+1\right)\left(m_{1}+m_{2}+n_{1}+n_{2}\right) !}{\Gamma(s+1) \Gamma\left(m_{1}-n_{1}+s+1\right) \Gamma\left(l-m_{1}-s+1\right)\left(l+m_{2}-k+2\right) !} \times \\
& \times \frac{\left(m_{2}-n_{2}-k+1\right)_{k}}{\left(l+m_{2}-k+1\right)_{k}\left(m_{2}-l-k\right)_{k}} \times \\
& \times t^{m_{1}+m_{2}+s+p-n_{1}-n_{2}-k+1}{ }_{2} F_{1}\left(\begin{array}{c}
l+m_{2}-k+1, m_{2}-l-k \\
m_{2}-n_{2}-k+1
\end{array} \mid t\right) .
\end{aligned}
$$

Для исследования сходимости произведения $\left\langle\boldsymbol{\Psi}_{1} \mid \boldsymbol{\Psi}_{2}\right\rangle$ применим следующее асимптотическое разложение для гипергеометрической функции [21]:

$$
\begin{aligned}
& { }_{2} F_{1}\left(\begin{array}{c}
a, b \\
c
\end{array} \mid t\right)=\frac{\Gamma(c) \Gamma(b-a)}{\Gamma(b) \Gamma(c-a)}(-t)^{-a}{ }_{2} F_{1}\left(\begin{array}{c|c}
a, 1-c+a & 1 \\
1-b+a & t
\end{array}\right)+ \\
& +\frac{\Gamma(c) \Gamma(a-b)}{\Gamma(a) \Gamma(c-b)}(-t)^{-b}{ }_{2} F_{1}\left(\begin{array}{c|c}
b, 1-c+b \\
1-a+b
\end{array} \mid \frac{1}{t}\right) .
\end{aligned}
$$

Таким образом, получаем

$$
\begin{aligned}
& \left\langle\boldsymbol{\Psi}_{1} \mid \boldsymbol{\Psi}_{2}\right\rangle=\frac{1}{\Gamma\left(m_{2}-n_{2}+1\right)} \sqrt{\frac{\Gamma\left(l-m_{1}+1\right) \Gamma\left(l-n_{1}+1\right) \Gamma\left(l-n_{2}+1\right) \Gamma\left(l+m_{2}+1\right)}{\Gamma\left(l+m_{1}+1\right) \Gamma\left(l+n_{1}+1\right) \Gamma\left(l-m_{2}+1\right) \Gamma\left(l+n_{2}+1\right)}} \times \\
& \times \sum_{s=0}^{l-m_{1}} \sum_{p=0}^{m_{1}+m_{2}+n_{1}+n_{2}} \sum_{k=1}^{m_{1}+m_{2}+s+p-n_{1}-n_{2}+1}(-1)^{m_{1}+m_{2}+n_{1}+n_{2}+p+k} \times \\
& \times \frac{\Gamma\left(l+m_{1}+s+1\right)\left(m_{1}+m_{2}+n_{1}+n_{2}\right) !}{\Gamma(s+1) \Gamma\left(m_{1}-n_{1}+s+1\right) \Gamma\left(l-m_{1}-s+1\right)\left(l+m_{2}-k+2\right) !} \times \\
& \times \frac{\left(m_{2}-n_{2}-k+1\right)_{k}}{\left(l+m_{2}-k+1\right)_{k}\left(m_{2}-l-k\right)_{k}} \times \\
& \times\left[(-1)^{l+m-k+1} \frac{\Gamma\left(m_{2}-n_{2}-k+1\right) \Gamma(-2 l-1)}{\Gamma\left(m_{2}-l-k\right) \Gamma\left(-n_{2}-l\right)} \times\right. \\
& \times t^{m_{1}+s+p-n_{1}-n_{2}-l}{ }_{2} F_{1}\left(\begin{array}{c|c}
l+m_{2}-k+1, l+n_{2}+1 & \frac{1}{t}
\end{array}\right)+ \\
& +(-1)^{m_{2}-l-k} \frac{\Gamma\left(m_{2}-n_{2}-k+1\right) \Gamma(2 l+1)}{\Gamma\left(l+m_{2}-k+1\right) \Gamma\left(-n_{2}-l\right)} \times \\
& \times t^{m_{1}+l+s+p-n_{1}-n_{2}+1}{ }_{2} F_{1}\left(\begin{array}{c|c}
m_{2}-l-k, n_{2}-l & \frac{1}{t} \\
2-2 l
\end{array}\right) .
\end{aligned}
$$

В этом выражении гипергеометрическая функция ${ }_{2} F_{1}$ может быть записана в виде степенного ряда по $1 / t$. Отсюда следует, что $\left\langle\boldsymbol{\Psi}_{1} \mid \boldsymbol{\Psi}_{2}\right\rangle \sim t^{m_{1}+l+s+p-M-n_{1}-n_{2}+1}$, а поскольку $M \rightarrow \infty$, то $\left\langle\boldsymbol{\Psi}_{1} \mid \boldsymbol{\Psi}_{2}\right\rangle$ сходится, когда $M>m_{1}+l+s+p-n_{1}-n_{2}+1$. 


\section{4. ЗАКЛЮЧЕНИЕ}

В настоящей работе приводится расширение метода группового усреднения посредством определения интегралов, задающих внутренние произведения для свободных полей, над однородными пространствами группы де Ситтера $S O_{0}(1,4) . N$-частичный случай рассмотрен над четырехмерным гиперболоидом $H^{4}$. Представляет интерес рассмотрение внутренних произведений, а также исследование сходимости получающихся интегралов над другими однородными пространствами (как симметрическими римановыми, так и неримановыми) группы $S O_{0}(1,4)$, такими как трехмерная вещественная сфера $S^{3}$, двумерная кватернионная сфера $S_{2}^{q}$ и групповое многообразие $\mathfrak{S}_{10}$ группы $S O_{0}(1,4)$. Исследованию этих вопросов будет посвящена следующая статья.

\section{Список литературы}

[1] П. А. М. Дирак, Принципъ квантовой механики, Физматгиз, М., 1960.

[2] M. Henneaux, C. Teitelboim, Quantization of Gauge Systems, Princeton Univ. Press, Princeton, 1992.

[3] N. Woodhouse, Geometric Quantization, Oxford Math. Monogr., Clarendon Press; Oxford Univ. Press, New York, 1980.

[4] J. R. Klauder, "Product representations and the quantization of constrained systems", $M a-$ тематическая физика. Проблемы квантовой теории поля, Сб. статей. К 65-летию со дня рождения академика Л.Д. Фаддеева, Тр. МИАН, 226, ред. А.А. Славнов, Е. Ф. Мищенко, Наука, М., 1999, 212-222, arXiv: quant-ph/9811051.

[5] H. B. G. S. Grundling, C. A. Hurst, Comm. Math. Phys., 98:3 (1985), 369-390.

[6] A. Ashtekar, Lectures on Non-perturbative Canonical Gravity, Adv. Ser. Astrophys. Cosmol., 6, World Sci., River Edge, NJ, 1991.

[7] A. Ashtekar, J. Lewandowski, D. Marolf, J. Mourão, T. Thiemann, J. Math. Phys., 36:11 (1995), 6456-6493.

[8] D. Marolf, "Refined algebraic quantization: systems with a single constraint", Symplectic Singularities and Geometry of Gauge Fields, Banach Center Publ., 39, Polish Acad. Sci., Warsaw, 1997, 331-344, arXiv: gr-qc/9508015.

[9] N. P. Landsman, J. Geom. Phys., 15:4 (1995), 285-319.

[10] M. A. Rieffel, Adv. Math., 13:2 (1974), 176-257.

[11] D. Marolf, Class. Quant. Grav., 12:5 (1995), 1199-1220, arXiv: gr-qc/9404053.

[12] A. Higuchi, Class. Quant. Grav., 8:11 (1991), 1983-2004.

[13] D. Giulini, D. Marolf, Class. Quant. Grav., 16:7 (1999), 2489-2505, arXiv: gr-qc/9902045.

[14] D. Marolf, I. A. Morrison, Class. Quant. Grav., 26:23 (2009), 235003, 28 pp., arXiv: 0810.5163.

[15] O. Yu. Shvedov, Ann. Phys., 302:1 (2002), 2-21, arXiv: hep-th/0111270.

[16] V. V. Varlamov, J. Phys. A, 40:1 (2007), 163-201, arXiv: math-ph/0604026.

[17] V. V. Varlamov, J. Phys. A, 37:20 (2004), 5467-5476, arXiv: math-ph/0308038.

[18] V. V. Varlamov, J. Phys. A, 39:4 (2006), 805-822, arXiv:math-ph/0507056.

[19] V. V. Varlamov, Internat. J. Modern Phys. A, 20:17 (2005), 4095-4112, arXiv: math-ph/0310051.

[20] А. П. Прудников, Ю. А. Брычков, О. И. Маричев, Интегралы и ряды. Дополнителъные главы, Наука, М., 1981.

[21] Г. Бейтмен, А. Эрдейи, Высшие трансцендентные функиии. т. 1: Гипергеометрическая функиия. Функиии Лежандра, Наука, М., 1965. 